\title{
DSC reveals variation in enthalpy associated with free water molecules in water-ethanol solution exposed to $x$-rays and magnetic field
}

\author{
Nirmal C. Sukul ${ }^{12}$, Tandra Sarkar ${ }^{1,3}$, Atheni Konar ${ }^{1,3}$, Mohamad Amir Sohel ${ }^{4}$, Asmita Sengupta ${ }^{4}$, \\ Anirban Sukul ${ }^{1}$ \\ ${ }^{1}$ Sukul Institute of Homeopathic Research, Santiniketan, West Bengal India; ${ }^{2}$ Department of Zoology, Visva- \\ Bharati University, Santiniketan, West Bengal, India; ${ }^{3}$ Centre for Healthcare Science and Technology, Indian \\ Institute of Engineering Science \& Technology, Shibpur, West Bengal, India; ${ }^{4}$ Department of Physics, Visva- \\ Bhartati University, Santiniketan, West Bengal, India \\ * Corresponding author: ncsukul@gmail.com.
}

Abstract

Aqueous ethanol is the standard medium for all drugs used in homeopathy. X-ray and Magnetis poli ambo are 2 homeopathic drugs prepared by exposure of aqueous ethanol to x-rays and static magnetic field, respectively. Mother tinctures (MT) were successively diluted with solvent 1:100 and succussed in several steps to prepare centesimal potencies $8 \mathrm{cH}, 14 \mathrm{cH}$ and $32 \mathrm{cH}$. The solvent was processed in the same way. Although identical in chemical composition ( 0.03 molar ethanol) and water content (96\%) these preparations like the Mother tinctures and three potencies of X-ray and Magnetis poli ambo exhibit different therapeutic pathological effects. Potency $8 \mathrm{cH}$ of each preparation was diluted with water to reach concentrations $4 \%, 20 \%, 40 \%$ and $80 \%$ ethanol. The aim of the study was to establish whether these potencies exhibited variation in free water molecules. Differential Scanning Calorimetry (DSC) of MT and potencies exhibited almost similar freezing and melting points, but they remarkably differed in freezing and melting enthalpy and free water molecules. The various dilutions of potency $8 \mathrm{cH}$ exhibited variation in enthalpies and free water molecules, being this variation independent of the amount of water added. We conclude that exposure of aqueous ethanol to x-rays and magnetic field, with subsequent dilution and agitation induces changes in the solvent involvipg re- ater poledes. All X-ray and Magnetis poli ambo potencies were analyzed by means of Raman spetroscbpy for free water molecules. The results were compared to the ones of DSC, being more or less similar.

Keywords: Homeopathic Potencies, X-ray, Magnetis poli ambo, DSC, Free Water Molecules

(C) International Journal of High Dilution Research.

Not for commercial purposes.

Cite as: Sukul NC, Sarkar T, Konar A, Sohel Amir Sengupta A, Sukul A. DSC reveals variation in enthalpy associated with free water molecules in water-ethanol solution exposed to x-rays and magnetic field. Int J High Dilution Res. 2018;17 (1):7 